\title{
Rapid Maxillary Expasion - An Exclusive Technique in Orthodontics
}

Sharath Kumar Shetty ${ }^{1}$, Sagar Desai ${ }^{2}$, Mahesh Kumar $Y^{3}$, Vijayananda K Madhur ${ }^{4}$, Brigit Alphonsa ${ }^{4}$

${ }^{1}$ Professor \& HOD, Department of Orthodontics and Dentofacial Orthopaedics, K. V. G. Dental College and Hospital, Sullia, Karnataka, India

${ }^{2}$ Post Graduate Student, Department of Orthodontics and Dentofacial Orthopaedics, K. V. G. Dental College and Hospital, Sullia, Karnataka, India

${ }^{3}$ Professor, Department of Orthodontics and Dentofacial Orthopaedics, K. V. G. Dental College and Hospital, Sullia, Karnataka, India

${ }^{4}$ Reader, Department of Orthodontics and Dentofacial Orthopaedics, K. V. G. Dental College and Hospital, Sullia, Karnataka, India

DOI: $10.36347 /$ sjds.2020.v07i12.007

| Received: 11.12.2020 | Accepted: 26.12.2020 | Published: 30.12 .2020

*Corresponding author: Sagar Desai

Abstract

Review Article

The dental discrepancy in transverse dimension can be due to arch length and the tooth material discrepancy i.e. narrow apical base of the jaw and lager teeth and could be due to lingual position of the upper posteriors or buccal position of the lower posteriors or combination. Arch expansion is an important part of clinician's armamentarium in the correction of transverse discrepancy [1]. There are many means and modes for expansion of arch, such as rapid, slow, surgically assisted, mini-screw anchored or simply by orthodontic tooth movement. Various types of removable or fixed expansion devices have been developed over the years.

Keywords: RME, Expansion, Exclusive Technique.

Copyright (C) 2020 The Author(s): This is an open-access article distributed under the terms of the Creative Commons Attribution 4.0 International License (CC BY-NC 4.0) which permits unrestricted use, distribution, and reproduction in any medium for non-commercial use provided the original author and source are credited.

\section{INTRODUCTION}

Arch expansion is an important part of clinician's armamentarium in the correction of transverse discrepancy [1]. The prime objective of arch expansion is to obtain coordination between maxillary and mandibular denture bases. The means of correcting a transverse, discrepancy can be via orthopaedic (skeletal), orthodontic (dental), or surgically assisted techniques [2]. The etiologic factors for transverse maxillo-mandibular discrepancy are congenital, iatrogenic, traumatic and developmental. The congenital presence of cleft palate restricts the normal development of maxilla thus causing maxillary hypoplasia. Moreover the surgical repair of cleft palate deformity by alveolar grafts leads to scarring of the palatal mucosa which further causes constriction of the maxillary arch. Hence this is best example for iatrogenic cause of transverse discrepancy. Similarly any traumatic injury caused during the normal development of maxilla and or the mandible can impede the growth and thus narrow the apical base [3]. The most ancient and effective treatment modality for elimination of transverse discrepancy is through expansion of arch. The prime objective of arch expansion is to achieve coordination between the maxillary and mandibular jaw bases.

\section{History}

A number of methods for the expansion of the maxilla were carried out by crude devices. These were mostly slow expansion and dated back from 1728 by Fauchard, Fox 1803, Delabarre 1819, Lefoulon 1839 \& 1841 , Shange 1841,Tomes 1848, Allen 1850, Whitel \& Westcott 1859 [7]. Wescott first reported the placement of mechanical forces on the bones of the maxilla in 1859 Wescott, 1859. He used two double-clasps separated by a telescopic bar to correct a crossbite in a 15-year-old girl. However, by the author's own admission the entire expansion procedure was slow and tedious and lasted several months.

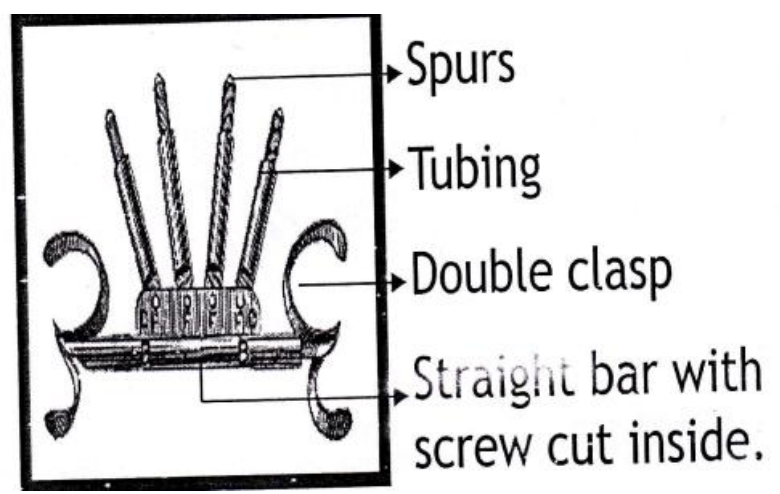

Angel was the first to use Rapid maxillary expansion device. He placed an appliance between the maxillary premolars of a 14 and half year old girl and widened her arch one quarter inch in two weeks. Goddard, in 1983, further standardized the palatal expansion protocol. In the year 1870, C. R. Coffin, Kinngsley's student, reported on a new design for an 
expansion appliance which still bears his name. Korkhaus and Andrew Hass, during 1950's reintroduced RME to the orthodontic community.

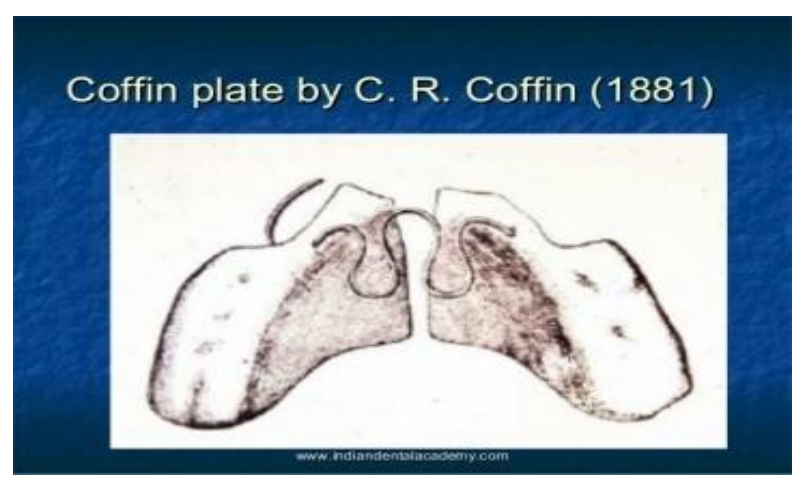

Anatomy

Palatine bones form an intimate relationship with maxilla to form complete hard palate (or) floor of nose and greater part of lateral wall of nasal cavity. It articulates anteriorly with maxilla through transverse palatal sutures and posteriorly through pterygoid process of rsthe sphenoid bone. The interpalatine suture joins the two palatine bones at their horizontal plates and continous as intermaxillary sutures. These sutures form the junction of three opposing pairs of bones: the premaxillae, maxilla, and the palatine. The entire forms mid-palatal suture [5].
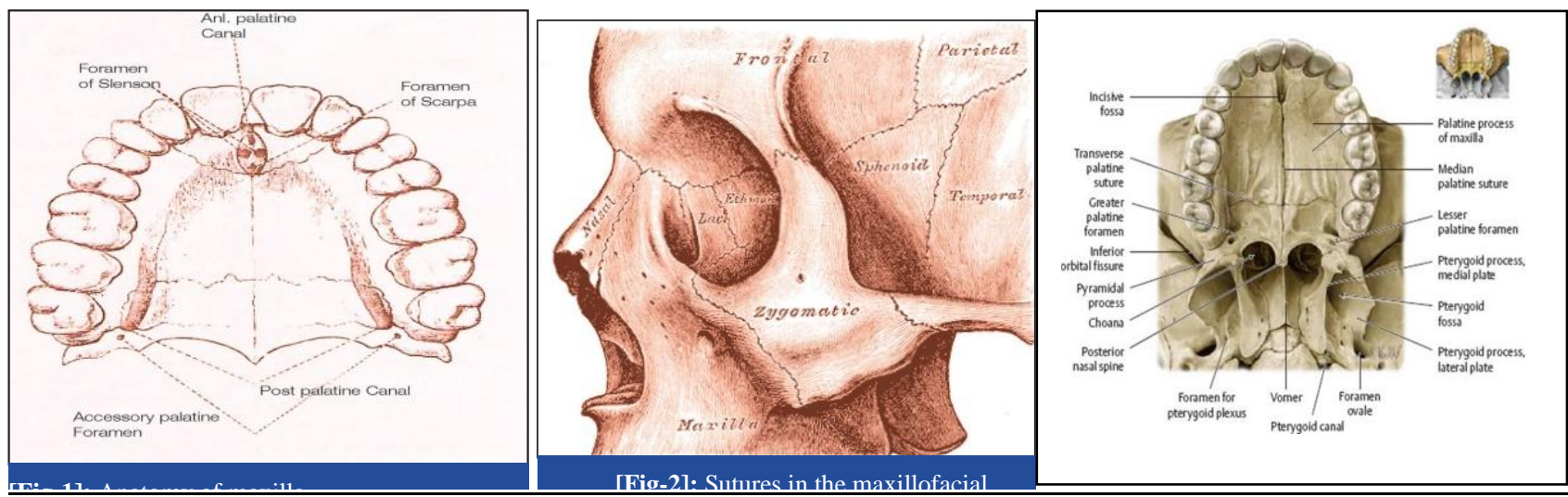

The interpalatine and intermaxillary sutue are collectively called mid palatal suture (MPS). It plays a key role in expansion of maxillary arch. Melsen 1975

1. Infancy -Y-shape

2. Juvenile -T-shape

3. Adolescence -Jigsaw Puzzle reports the histological feature of MPS from infant to adult.
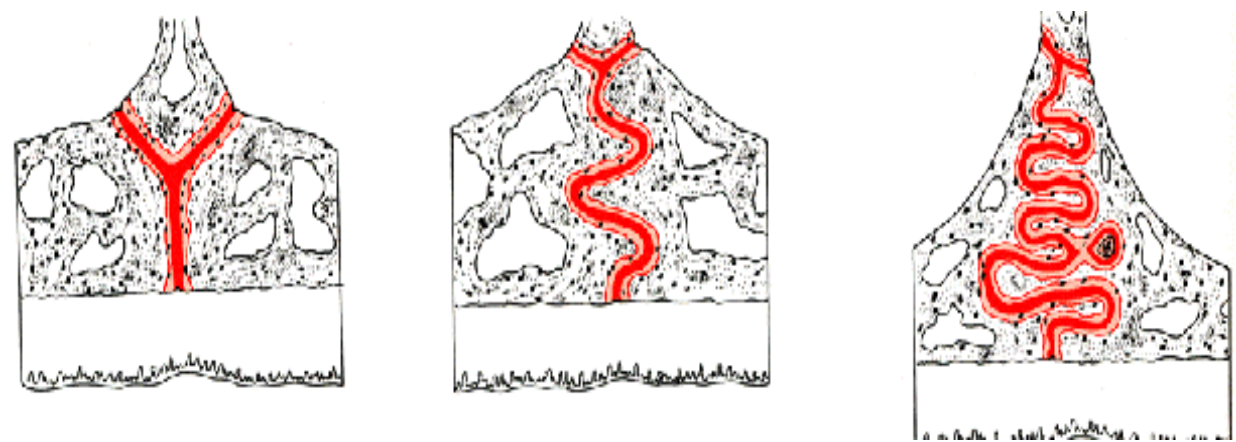

As sutural patency is vital to R.M.E, it is important to know when does the suture closes by synostosis [2] and on an average 5\% of suture in closed by age 25 yrs. Earliest closure occurs in girls aged 15 yrs. Greater degree of obliteration occurs posteriorly than anteriorly. Ossification comes very late anterior to incisive foramen - this is important when planning surgical freeing in late instances of RME [3].

Factors to be considered before expansion Important factors to be considered in Rapid Maxillary Expansion

1. Rate of Expansion: By expanding at the rates of
$0.3-0.5 \mathrm{~mm}$ per day, active expansion is completed in 2-4 weeks, leaving little time for the cellular response of osteoclasts and osteoblasts seen in slow expansion.

2. Form of Appliance: As the thrust is delivered to the teeth at the inferior free borders of the maxilla, expansion must reach to the basal portions. The form of appliance will play an important role in this effort, according to its rigidity or flexibility, i.e. anchorage or control of tipping.

3. Age and Sex of the patient: The increasing rigidity of the facial skeleton with advancing age restricts bony movements remote from the appliance 
of expansion, which differs in both sexes.

4. Discrepancy between maxillary and mandibular first molars \& bicuspid width is $4 \mathrm{~mm}$ or more RME indicated.

5. Severity of cross bite i.e number of teeth involved.

6. Initial angulation of molars or premolars: When the maxillary molars are buccally inclined, conventional expansion will tip them further into the buccal musculature and if the mandibular molars are lingually inclined, the buccal movement to upright them will increase the need to widen the upper arch.

7. Assessment of roots of deciduous tooth

8. Physical availability of space for expansion.

9. Nasal obstruction: All patients considered for RME should be examined for nasal obstruction and, if obstruction is found, they should be referred to an otolaryngologist before orthodontic treatment.

10. Medical history: Since the efficacy of maxillary expansion depends on suture patency and the flexibility of craniofacial compelex to adapt to mechanical changes hence medical conditions altering these should be considered.

11. Meatbolic disorders: Many metabolic disorders are found associated with suture synotoses which include hyperthyroid- ism, hypophosphatemic vitamin D-resistant rickets, and mucopolysaccharidoses and mucolipidoses. These disorders are mostly associated with bone metabolism. Maxillary expansion would be futile even in young patients if they are suffering from any of these diseases.

12. Periodontal Type: It is essential to record the thickness of the gingival tissues during clinical evaluation of the periodontium. This is especially important because a thin and delicate gingiva might be prone to recession after traumatic, surgical, or inflammatory injuries [4].

13. Mucogingival Health: Orthodontic tooth movement has significant effect on the mucogingival tissues and hence it is important to asses the periodontal health of the patient before performing OME.

\section{Indication for RME}

Haas (1965) [21] gave the following six conditions as indications for expansion.

1. Cases of real and relative maxillary deficiency with posterior cross bite. A real deficiency is characterized by compression of the maxilla when compared to the rest of the facial bones and mandible i.e. the maxilla is definitely undersized. A relative deficiency exists when the maxilla appears to be of normal size when compared with adjacent craniofacial bones but the mandible is mildly or even moderately over sized.

2. Cases of nasal stenosis - usually characterized by full time mouth breathing and a constricted nasal aperture with the conchae literally compressed against the Septum.
3. All types of Class III cases i.e., pseudo, dental, and skeletal. In approximately 3 weeks there is correction of cross bite (anterior and posterior) and improvement of denture base relationship.

4. Mature cleft palate cases with collapsed maxilla.

5. Antero-posterior maxillary deficiency cases. These are cases with negative ANB angles, a negative point A to facial plane, or a negative wits analysis which would benefit from maxillary protraction.

6. A prerequisite to such activity would be expansion to loosen the maxilla to facilitate protraction. Selected arch length problems in mature good morphogenetic skeletal pattern especially some patients of 14-16 years where extraction could lead to flattering of profile.

GRAY (1975) [19] recognizes the medical indications for expansion as a combination of the following:

1. Poor nasal airway

2. Septal deformity

3. Recurrent ear, nasal or sinus infection

4. Allergic rhinitis

5. Asthma

6. Prior to septoplasty - In the narrow compressed nose, RME widens then enabling a septoplasty to be a reasonable proposition.

\section{CONTRA INDICATIONS}

1. Poor patient co-operation.

2. Single tooth in cross bite.

3. Some operators set an upper age limit, ie, the age when synostosis has occurred. But with adjunctive surgery this is surmounted.

4. Normal buccal occlusion. However if there an overriding medical reasons of a respiratory nature for expansion, we have to give priority to this and correct.

5. If there is a medical reason for expansion but with "over bite" (i.e. maxillary teeth completely outside mandibular teeth) then it is a definite contraindication.

6. Skeletal asymmetry of maxilla or mandible and adults with severe anteroposterior and unilateral skeletal discrepancies. Expansion can be done if it is to be followed with orthognathic surgery.

7. Anterior open bite, steep mandibular plane and convex profile are not generally suited for expansion. This is surmounted using bonded appliances with posterior bite blocks.

8. If expansion is necessary in a long face-open bite patient it is advantageous to use a bonded expansion appliance that incorporates bite blocks. This controls the increase in face height that otherwise can be expected.

9. Periodontally weak teeth.

\section{Types}

RME APPLIANCES

1. Removable appliances

2. Fixed appliances 
i. Tooth borne

ii. Tooth and tissue borne-

1. Banded RME a) Hass, b) Isaacson, c) Hyrax and d) Derichsweiller

2. Bonded RME - a) Acrylic splints, and b) Cast metal splints.

\section{Diagnostic Aids}

Case History, Clinical examination, study models, radiographs - maxillary occlusal, P.A. cephalogram

1. A radiologically visible midpalatal suture corresponds histologically to a predominantly straight running oronasal suture, which projects largely into the saggital X-ray path

2. Radiological invisible suture corresponds histologically to a relatively large area of interdigitation, an oblique running suture course in relation to X-ray path or bone structures projecting above the suture course. Percentage of suture obliteration to be expected is also low in this group.

3. A radiologically invisible suture is not histologically equivalent of fused suture.

\section{Biomechanical Requirements}

Timms 1981 has listed the criteria based on the biomechanical requirements of expansion device by which an appliance design may be attempted by an objective approach. The list in descending order of importance is as follows [5]:

1. Rigidity it is one of the most important requirements of the appliance.

2. Tooth utilization-load distribution- as many teeth as possible should be included.

3. Retention-increase in number of teeth utilized with their retentive areas will increase the retention of the appliance.

4. Expansion screw-it should of sufficient length to complete the expansion without interruption.

5. Economic of time and material.

6. Hygienic.

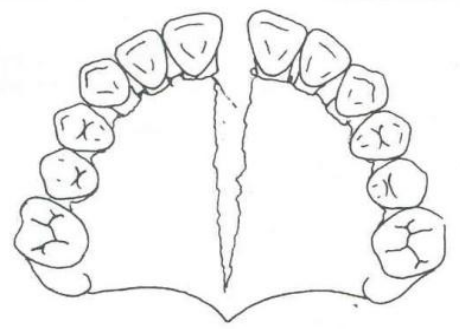

\section{Palatal Vault}

The palatine processes of the maxilla were lowered as a result of the outward tilting of the maxillary halves, also the palatal vault height decreased significantly during RME. Palatal height returned to pretreatment values one year after expansion and
Cap splints should be fixation of choice, especially where rigidity is important $\&$ bands have their place, where there are difficulties in retention.

Instructions to patients have been classified into 3 age groups [6].

1. Till age 15 years- $180^{\circ}$ daily rotation can be met with turn of $90^{\circ}$ both morning \& evening. Patient recalled after one week.

2. Age 15 to 20 years -Increasing resistance for maxillary separation may cause a force buildup \& pain to patients in this age group with turns of $90^{\circ}$. Patients are asked to return after one week.

3. Above age of 25 years -The mid palatal suture often is opened surgically which relives much of the tension. Here it may not be necessary to reduce the overall rate of expansion in these patients. Revisit within 34 days. Pain to be reviewed during active RME, before continuing with patient management during subsequent visits.

\section{EFFECTS OF EXPANSION ON THE MAXILLARY COMPLEX}

When an appropriate reciprocal force is applied to the maxillary teeth and alveolar process laterally across the palate exceeds the limit for orthodontic tooth movement and overcomes the bioelastic strength of sutural elements, it results in rapid separation of the two halves of the maxilla i.e. a rapid disarticulation occurs along their common midpalatal suture.

\section{Maxillary Halves}

It is seen that the two halves of the maxilla rotated in both the sagittal and frontal planes. The maxilla was found to be more frequently displaced downward and forward [8]. Haas suggested when the midpalatal suture opens, the maxilla always moves forward and downward. Skeletal changes in vertical and anterior displacement of maxilla with bonded rapid palatal expansion appliances using the lateral cephalograms showed that downward and anterior displacement of the maxilla may be minimized or negated with the use of the bonded appliances.

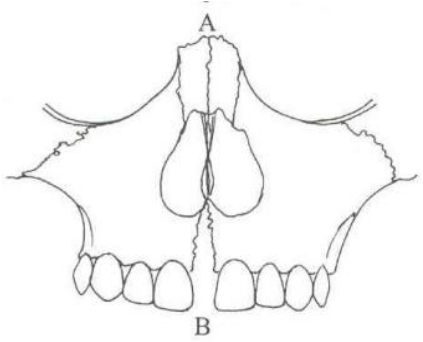

increased an average of $0.5 \mathrm{~mm}$ two years after treatment.

\section{Alveolar Process}

It has been seenin studies that sincebone is resilient, lateral bending of the alveolar processes occurs early during RME [6]. 


\section{Biological Response}

The immediate effect of applying force to the suture results in trauma. Small, localized tears occurred within the suture from the localized blood vessels. These small defects were filled with exudate, a few extravasated red blood cells, scattered filaments of fibrin and a few fine collagen fibrils [9]. A transient polymorph response was noted in the region of the defects in the first 12 hours and thereafter was not seen again. Following the polymorph response, an influx of macrophages and pioneer fibroblasts into the defect occurred by 24 hours. Within 3 to 4 days, bone formation had begun at the margins of the suture achieved by the pre-existing and undamaged osteoblasts. These formed successive lamellae along the suture margin. The collagen fibers and cells were aligned transversely across the suture corresponding to levels of tension. New bone formation now occurred along the same axis as trabeculae formed at right angles to the lamellae deposited initially at the suture margins. With diminution and cessation of the expansion force ( 2 to 3 weeks), remodeling of both the bone and the suture occurred by the osteocytic and fibrocytic cell series until normal sutural dimensions were achieved. The mineral content within the suture rose rapidly during the first month after the completion of suture opening. The mineral content in the bone beside the suture decreased rapidly in the first month but returned to its initial level within 3 months [10].

\section{Change in Anterior Teeth}

From the patient's point of view, one of the most spectacular changes accompanying RME is the opening of a diastema between the maxillary central incisors. It is estimated that during active suture opening, the incisors separate approximately half the distance the expansion screw has been opened. Following this separation, the incisor crowns converge and establish proximal contact. If a diastema is present before treatment, the original space is either maintained or slightly reduced. The mesial tipping of the crowns is due to the elastic recoil of the transseptal fibers. Once the crowns contact, the continued pull of the fibers causes the roots to converge toward their original axial inclinations. This cycle generally takes about 4 months.

\section{Change in Posterior Teeth}

With the initial alveolar bending and compression of the periodontal ligament, there is a definite change in the long axis of the posterior teeth. Teeth show buccal tipping and believed to extrude to a limited extent [11].

\section{Change in the Mandible}

The greatest increase in uprighting of the buccal segments was in the bonded RME case for the lower arch. RME could lead to a concurrent expansion of the lower arch as much as $4 \mathrm{~mm}$ in inter-canine width and $6 \mathrm{~mm}$ in inter-molar width [12].

\section{Change in adjacent Facial structures}

All craniofacial bones directly articulating with the maxilla were displaced except the sphenoid bone. The cranial base angle re- mained constant. Displacement of the maxillary halves was asym- metric, the sphenoid bone, and not the zygomatic arch, was the main buttress against maxillary expansion.

\section{Changes in Soft Tissue}

According to a study by, the effect of RME on soft tissues, the nose tip and soft tissue Point A followed the anterior movement of the maxilla and maxillary incisors. Nihat Kilic et al., [17] concluded in their study that the soft tissue facial angle decreases and the $\mathrm{H}$ angle and profile convexity increases after RME. Also the $\mathrm{H}$ angle and profile convexity were statistically significant for their study [14].

\section{Recent Methods in Expansion}

The most recent method used in the treatment of maxillary transverse deficiency (MTD) is Surgically Assisted Rapid Palatal Expansion (SARPE). Orthopedic Maxillary expansion (OME), in mature patient has been found associated with laterally tipping of teeth, extrusion, periodontal membrane compression, buccal root resorption, alveolar bone bending, fenestration of buccal cortex, palatal tissue necrosis, pain and instability of expansion. Because of the complications of OME SARPE has been recommended as a treatment of choice. Recent advances in molecular biology has identified the underlying mechanism in suture fusion which is an important criteria for successful long term maxillary expansion. Increased rate of cell numbers and cell differentiation can cause the formation of a bony obliteration in between the sutures.

\section{RETENTION \& RELAPSE}

The objective of retention here is to hold the expansion while all other forces generated by the expansion have decayed away. Studies by Krebs 1964 and Timms 1976 shows that 5 years may pass before ultimate stabilization is reached, although the amount of relapse during the last two year being very small. The first 2 and half year is the critical phase and it is essential in the first three months, that fixed appliance acts as the retentions appliance. Following the removal of fixed appliance, the mouth is left without any appliance for a few days (2-4) to permit the recovery of palatal mucosa. The retention phase here is given with 4 Adam's cribs ( 2 on molar and 2 on premolars). In mixed dentition the fixed retention is longer about 6 months and the retention plate is given with only 2 Adam's Cribs. In an ideal state, the maxillary dental arch will be held now with its palatal cusps occluding with the mandibular buccal cusps. When the stress and strains are eliminated the retention plate can be discarded and the teeth can tilt inward setting into their natural occlusion under environmental influences. If the retention plate is not worn for any appreciable period of 
time, there may be sufficient relapse; so as to prevent the proper placement of the appliance.

\section{CONCLUSION}

Arch expansion is one of the oldest clinical armamentarium of the orthodontist in the correction of transverse discrepancy. The prime objective of arch expansion is to obtain interarch coordination between the denture bases. Through expansion intraarch space is gained which helps in correcting the tooth size -arch length discrepancy. Hence mild to moderate crowding correction can be corrected through non extraction.

Rapid maxillary expansion has the effect on the maxilla, mandible, periodontal tissue, pulp as well as circummaxillary sutures, nasal cavity, adjacent facial bones. Hence it is helpful for an individual with breathing difficulties due to narrow nasal cavity. Debate is being done on the stability and progress of expansion since the day the theory of expansion has been put forward. Despite this, considerable success has been achieved by expansion. With patient cooperation, expansion of the dental arches especially during the mixed dentition period has yielded good result. As with any form of therapy there are contraindications, occasional unfavorable responses and at times partial success. Nevertheless when properly used, expansion is of great value in treating malocclusion.

\section{REFERENCE}

1. Jeffrey L, Berger C. Photographic analysis of facial changes associated with maxillary expansion. Am J orthod Dentofacial orthop. 1999; 116:563-71.

2. William R. Profitt Contemporary orthodontics, 6th edition.

3. Gray, Henry. Anatomy of the Human Body. Philadelphia; Lea \& Febiger, 1918; Bartleby.com, 2000.

4. Melson B. Palatal growth study on human autopsy material: A histologic micro radiographic study. Am J Orthod. 1975; 68:42-54.

5. Persson M, Thilander B. Palatal suture closure in man from 15 to 35 years of age. Am J Orthod. 1977;72:42-52

6. Bjork A, Skieller V. Growth in width of the maxilla by the implant method. Scand J Plast Reconst Surgery. 1974; 8-22-33.
7. Suri and Taneja. Surgically assisted rapid palatal expansion: A literature review, American Journal of Orthodontics and Dentofacial Orthopedics. 133(2):776-780.

8. Haas AJ. Rapid Expansion of the Maxillary Dental Arch and Nasal Cavity by Opening the Midpalatal Suture, Angle Ortho. 1961; 31:73-89.

9. Isaacson RJ, Ingram AH. Forces produced by rapid maxillary expansion. Part II. forces present during treatment. Angle Orthod. 1964; 34:261-9.

10. Zimring JF, Isaacson RJ. Forces produced by rapid maxillary expansion. III. Forces present during retention. Angle Orthod. 1965; 35:178-86.

11. Haas AJ. The treatment of maxillary deficiency by opening the midpalatal suture, Angle Orthodont. 1965; 35: 200-217.

12. Ten Cate AR, Freeman E, Dickinson JB. Sutural development: structure and its response to rapid expansion. Am J Orthod. 1977; 71:622-36.

13. Ekstrm. C, Henrickson CO and Jeensen R. Mineralization in the midpalatal suture after orthodontic expansion. Am J Orthod. 1977; 71:449-55.

14. Hicks EP. Slow maxillary expansion: a clinical study of the skeletal vs dental response in low magnitude force. Am J Orthod . 1978; 73:121-41.

15. Sandstrom RA, Klaper L, Papaconstantinou S. Expansion of the lower arch concurrent with rapid maxillary expansion. Am J Orthod. 1988; 94:296302.

16. Doruk C. Comparison of nasal volume changes during rapid maxillary expansion using acoustic rhinometry and computed tomography, European Journal of Orthodontics, 2007:29:251-255.

17. Nihat K. Effects of rapid maxillary expansion on Holdaway soft tissue measurements, European Journal of Orthodontics, 1998; 30(3):239-243.

18. Vardimon AD, Graber TM, Pitarn S. Repair process of external root resorption subsequent to palatal expansion treatment. Am J Orthod. 1993; 103:120130.

19. Gray LP. Results of 310 cases of RME selected for medical reasons. J Laryngol Otol. 1975; 89(6):601-14.

20. Hass AJ. The treatment of Maxillary deficiency by opening the midpalatal suture. Angle Orthod. 1965; 35:200-2. 